\title{
The fractal geometry of Hartree-Fock
}

Friethjof Theel, Antonia Karamatskou, and Robin Santra

Citation: Chaos 27, 123103 (2017);

View online: https://doi.org/10.1063/1.5001681

View Table of Contents: http://aip.scitation.org/toc/cha/27/12

Published by the American Institute of Physics 


\title{
The fractal geometry of Hartree-Fock
}

\author{
Friethjof Theel, ${ }^{1, a)}$ Antonia Karamatskou, ${ }^{1,2,3, b)}$ and Robin Santra ${ }^{1,2,3, c)}$ \\ ${ }^{1}$ Department of Physics, University of Hamburg, Jungiusstrasse 9, 20355 Hamburg, Germany \\ ${ }^{2}$ Center for Free-Electron Laser Science, DESY, Notkestrasse 85, 22607 Hamburg, Germany \\ ${ }^{3}$ The Hamburg Centre for Ultrafast Imaging, Luruper Chaussee 149, 22761 Hamburg, Germany
}

(Received 25 August 2017; accepted 15 November 2017; published online 7 December 2017)

\begin{abstract}
The Hartree-Fock method is an important approximation for the ground-state electronic wave function of atoms and molecules so that its usage is widespread in computational chemistry and physics. The Hartree-Fock method is an iterative procedure in which the electronic wave functions of the occupied orbitals are determined. The set of functions found in one step builds the basis for the next iteration step. In this work, we interpret the Hartree-Fock method as a dynamical system since dynamical systems are iterations where iteration steps represent the time development of the system, as encountered in the theory of fractals. The focus is put on the convergence behavior of the dynamical system as a function of a suitable control parameter. In our case, a complex parameter $\lambda$ controls the strength of the electron-electron interaction. An investigation of the convergence behavior depending on the parameter $\lambda$ is performed for helium, neon, and argon. We observe fractal structures in the complex $\lambda$-plane, which resemble the well-known Mandelbrot set, determine their fractal dimension, and find that with increasing nuclear charge, the fragmentation increases as well. Published by AIP Publishing. https://doi.org/10.1063/1.5001681
\end{abstract}

In the present work, we provide a new way of looking at the Hartree-Fock method, a widely used computational method for addressing the quantum-mechanical many-electron problem. A complex coupling strength $\lambda$ is introduced, and the convergence behavior of the Hartree-Fock iteration is analyzed in the complex plane for various noble gas atoms. Thus, we treat the iterative Hartree-Fock method as a dynamical system, with similarities to the construction of the Mandelbrot set. The investigation of the convergence behavior depending on the $\lambda$ parameter reveals interesting structures of the Hartree-Fock sets which strongly resemble fractals and allow for the definition of a fractal dimension. We find that the fragmentation of these sets increases with increasing nuclear charge. Since many-body perturbation theory builds on mean-field theories such as Hartree-Fock, analyzing the mathematical properties of Hartree-Fock iterations in the complex $\lambda$ plane is a scientifically well-motivated and new question. Therefore, our study can inspire new investigations towards performing many-body perturbation theory calculations.

\section{INTRODUCTION}

Describing the electronic wave function of atoms and nuclei adequately is of particular importance in physics and in chemistry. ${ }^{1-3}$ A widely used method for calculating atomic and molecular orbitals is the Hartree-Fock (HF) method. $^{4-10}$ The HF method is used for many-electron systems, such as molecules, or in nuclear physics for a manybody problem involving protons and neutrons. ${ }^{11-13}$ It is an iterative procedure based on the variational principle for

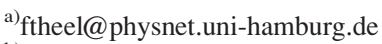

b) antonia.karamatskou@cfel.de

c)robin.santra@cfel.de
}

finding the ground-state energy of a system. The HF method gains further importance by providing a starting point for many-body methods. ${ }^{14-16}$ An example is the Møller-Plesset (MP) Perturbation Theory, ${ }^{17}$ which was developed to systematically capture electron-correlation effects.

Stillinger ${ }^{18}$ and Sergeev and Goodson ${ }^{19}$ investigated the convergence behavior of MP Perturbation Theory. To do so, they artificially introduced a complex factor $\lambda$ in front of the MP perturbation Hamiltonian $\hat{H}_{1}$

$$
\begin{gathered}
\hat{H}(\lambda)=\hat{H}_{0}+\lambda \hat{H}_{1}, \\
\hat{H}_{0}=\hat{T}+\hat{V}^{(\text {ne })}+\hat{V}^{(\mathrm{scf})}, \\
\hat{H}_{1}=\hat{H}_{\text {phys }}-\hat{H}_{0}=\hat{V}^{(\mathrm{ee})}-\hat{V}^{(\mathrm{scf})} .
\end{gathered}
$$

Here, $\hat{H}_{0}$ contains the electron kinetic operator $\hat{T}$, the electron-nucleus Coulomb potential $\hat{V}^{(\text {ne) }}$, and a mean-field potential $\hat{V}^{\text {(scf) }}$. The operator $\hat{H}_{\text {phys }}$ is related to the physical, nonrelativistic Hamiltonian with an electron-electron interaction potential $\hat{V}^{(\mathrm{ee})}$. In the case of $\lambda=1$, Eq. (1) reproduces $\hat{H}_{\text {phys }}$ and describes the physical system. With the Hamiltonian $\hat{H}(\lambda)$ from Eq. (1), the ground-state energy $E(\lambda)$ is expanded as a power series in $\lambda$. For some systems, $E(\lambda)$ is nonanalytic within the unit circle in the complex $\lambda$ plane. The consequence is that only for parameters of $\lambda$ with an absolute value smaller than the absolute value of the singular point, it is ensured that $E(\lambda)$ is analytic and its power series converges. Especially, the energy for $\lambda=1$ might not converge within MP Perturbation Theory.

In order to improve the convergence properties of the HF method, several more advanced methods were developed, such as Broyden's method, ${ }^{20-22}$ and were used in various fields of physics. However, here, we do not put the focus on improving the HF method itself, but rather we want to 
investigate the convergence behavior of the simple restricted closed-shell HF method for the noble gas atoms helium, neon, and argon. The procedure is similar to the one described above. We use the original HF Hamiltonian for an $N$-electron atom, implementing a complex factor $\lambda$ to control the magnitude of the self-consistent-field (SCF) potential, which is a mean-field potential generated by the Coulomb interaction among the $N$ electrons. From Eq. (1), it is obvious that when the complex control parameter $\lambda$ is varied in the complex plane, so is the electronic coupling-strength. For every $\lambda$, we check whether the HF iteration converges or not, thereby determining the set of parameters $\lambda$ for which the HF iteration converges in the complex coupling-strength plane. In the following, we will call the pattern of the entirety of converged $\lambda$ points the self-consistent-field (SCF) set. Note that the present methodology to investigate the convergence behavior of such a system only presupposes the existence of a mean-field, as, for example, in the restricted HF, which also exists for open shell atoms. In fact, the same method may be applied to any system to which Hartree-Fock can be applied, i.e., not only atoms but also molecules and solids.

The HF method is an iterative process, and therefore, we consider it as a time-dependent discrete dynamical system, where the solution of the previous step is the basis for the next step. Dynamical systems are well known in mathematics; one prominent example is chaos theory. ${ }^{23}$ The difference between most dynamical systems and the HF method is that HF iterates a set of functions in each step, whereas common dynamical systems iterate one complex number. This circumstance makes the problem of the convergence behavior of HF even more complex and unpredictable. However, some "simple" dynamical systems, such as the Mandelbrot set, ${ }^{24-26}$ can produce complex fractal structures when the control parameter is varied over the complex plane. This interesting behavior provides excellent motivation to investigate whether the SCF set exhibits fractal geometries too.

In Sec. II, the HF method and basic mathematical aspects of fractals are introduced. In addition, numerical aspects that we use in the calculations are presented. Section III contains the results of the analysis. Apart from showing the SCF set in the complex coupling-strength plane and results for the values of the fractal dimension for helium, neon, and argon, we examine the orbitals of helium and neon in greater detail. Section IV concludes this work with a summary and a brief outlook.

\section{THEORETICAL BACKGROUND AND METHODS}

This section gives an overview of the physical and mathematical methods we use in the calculations. First, we introduce the HF method without the complex factor $\lambda$. Then, we implement the factor $\lambda$ in the HF Hamiltonian and connect the idea of the resulting modified HF method with discrete dynamical systems. In addition, we discuss the properties of the modified HF method. Finally, we present the technique we use for estimating the fractal dimension of an arbitrary set of points and discuss the symmetry properties of the SCF set.

\section{A. Hartree-Fock approximation}

The HF method is an ab initio calculation based on the variational principle. ${ }^{27,28}$ Its purpose is to find the groundstate orbitals for an $N$-electron system, for which the energy is minimized.

The one-electron HF Hamiltonian $\hat{f}$ for a closed-shell atom is

$$
\hat{f}\left(\mathbf{r}_{i}\right)=\hat{h}\left(\mathbf{r}_{i}\right)+\hat{V}^{\text {(scf) }},
$$

with the core Hamiltonian $\hat{h}\left(\mathbf{r}_{i}\right)$ for the $i$ th electron and the SCF potential $\hat{V}^{\text {(scf) }}$, which consists of a static mean-field potential generated by the electron-electron interaction among all electrons

$$
\hat{V}^{(\text {scf) }}=2 \hat{J}-\hat{K},
$$

with the Coulomb operator $\hat{J}$ and the exchange operator $\hat{K}$. The Coulomb operator is local and affects every electron. In contrast to the Coulomb operator, the exchange operator is nonlocal and possesses no classical potential. $\hat{K}$ affects only electrons with the same spin polarization and therefore couples $N / 2$ electron pairs. If $\hat{f}$ is applied to the spatial orbital $\varphi_{i}$, we obtain an eigenvalue problem with the $i$ th orbital energy $\epsilon_{i}$

$$
\hat{f} \varphi_{i}=\epsilon_{i} \varphi_{i} .
$$

Expanding $\varphi_{i}$ in a basis set $\left\{\chi_{\rho}\right\}$ with the expansion coefficients $C_{\rho i}$, we obtain

$$
\varphi_{i}=\sum_{\rho=1}^{W} C_{\rho i} \chi_{\rho} .
$$

Using this basis set, we represent the Fock matrix $\mathbf{F}$, which we will also refer to as HF Hamiltonian, as

$$
F_{\rho \sigma}=\int \mathrm{d}^{3} r \chi_{\rho}^{*}(\mathbf{r}) \hat{f}(\mathbf{r}) \chi_{\sigma}(\mathbf{r}) .
$$

Assuming that the basis set is orthonormal, it follows that the algebraic eigenvalue equation can be written as

$$
\mathbf{F C}=\mathbf{C d i a g}\left(\epsilon_{1}, \ldots, \epsilon_{W}\right),
$$

where $\mathbf{C}$ is a $W \times W$ matrix that contains in its columns the eigenvectors of the HF Hamiltonian $\mathbf{F}$ and $\left\{\epsilon_{1}, \ldots, \epsilon_{W}\right\}$ is the spectrum of $\mathbf{F}$. Every column $\mathbf{C}_{i}$ refers to a spatial orbital $\varphi_{i}$. Equation (9) is a time-independent Schrödinger equation and is called Roothaan equation. ${ }^{29}$ The HF Hamiltonian $\mathbf{F}$ can be presented as

$$
\mathbf{F}=\mathbf{H}^{(\text {core })}+\mathbf{V}^{(\text {scf })}
$$

Here, $\mathbf{H}^{(\mathrm{core})}$ is the core Hamiltonian and written in the basis $\left\{\chi_{\rho}\right\}$

$$
\begin{aligned}
H_{\rho \sigma}^{(\text {core })} & =T_{\rho \sigma}+V_{\rho \sigma}^{(\mathrm{ne})} \\
& =\int \mathrm{d}^{3} r \chi_{\rho}^{*}(\mathbf{r})\left[-\frac{1}{2} \nabla^{2}-\frac{Z}{r}\right] \chi_{\sigma}(\mathbf{r}),
\end{aligned}
$$


with the charge $Z$ of the nucleus and the distance $r$ between the electron and the nucleus. Throughout, we will assume $\mathbf{H}^{\text {(core) }}$ to be real and symmetric. Also, the SCF potential operator $\hat{V}^{\text {(scf) }}$ is represented in the basis set $\left\{\chi_{\rho}\right\}$, so that we can write the operator as the matrix $\mathbf{V}^{\text {(scf) }}$. Consequently, we can write the Coulomb and exchange matrices

$$
\begin{aligned}
\left\langle\chi_{\rho}|\mathbf{J}| \chi_{\sigma}\right\rangle & =\sum_{i} \sum_{\mu \nu} C_{\mu i} C_{\nu i}(\rho \mu \mid \sigma \nu), \\
\left\langle\chi_{\rho}|\mathbf{K}| \chi_{\sigma}\right\rangle & =\sum_{i} \sum_{\mu \nu} C_{\mu i} C_{\nu i}(\rho \mu \mid \nu \sigma),
\end{aligned}
$$

with the double integral in (1212) notation

$$
(\rho \mu \mid \sigma \nu)=\int \mathrm{d}^{3} r \int \mathrm{d}^{3} r^{\prime} \chi_{\rho}^{*}(\mathbf{r}) \chi_{\mu}^{*}\left(\mathbf{r}^{\prime}\right) \frac{1}{\left|\mathbf{r}-\mathbf{r}^{\prime}\right|} \chi_{\sigma}(\mathbf{r}) \chi_{\nu}\left(\mathbf{r}^{\prime}\right) .
$$

Upon insertion of Eqs. (12) and (13) into Eq. (10), the nonlinear dependence of $\boldsymbol{F}$ on $\boldsymbol{C}$ becomes apparent. When later introducing a complex parameter $\lambda$ in the HF method, $\boldsymbol{C}$ will become complex. We will then continue to use Eqs. (12) and (13), i.e., we will not take the complex conjugate of $C_{\mu i}$. We further assume throughout that the integrals $(\rho \mu \mid \sigma \nu)$ are all real. These things, combined with the assumption that $\mathbf{H}^{\text {(core) }}$ is realsymmetric, ensure that the Fock matrix is symmetric. We will see in Sec. II D that this choice has an impact on the symmetry of the SCF set. The choice made for the $C_{\mu i}$ in Eqs. (12) and (13) is motivated by non-Hermitian quantum mechanics. ${ }^{30}$ In essence, as a consequence of the symmetry of $\mathbf{F}$, ensured by Eqs. (12) and (13), we may assume that the eigenvector matrix $\boldsymbol{C}$ of $\boldsymbol{F}$ satisfies the orthonormality condition

$$
\boldsymbol{C}^{\top} \boldsymbol{C}=\mathbb{1},
$$

where $\mathbb{1}$ is the identity matrix. For non-Hermitian $\boldsymbol{F}$, one cannot ensure $\boldsymbol{C}^{\dagger} \boldsymbol{C}=\mathbb{1}$, particularly not by replacing $C_{\mu i}$ in Eqs. (12) and (13) with $C_{\mu i}^{*}$.

Now, since the HF method is iterative, it is convenient to introduce as an index the number $n$ of iteration steps. Together with the $j$ th eigenvector $\mathbf{C}_{j}$, the Roothaan equation can be written as

$$
\sum_{\nu} F_{\mu \nu}^{(n)}\left(\mathbf{C}^{(n-1)}\right) C_{\nu j}^{(n)}=\epsilon_{j}^{(n)} C_{\mu j}^{(n)}
$$

Due to the fact that the $n$th HF Hamiltonian depends on the $(n-1)$ st eigenvectors, we have to make an initial guess $\mathbf{C}^{(0)}$ to start the iteration. We will assume $\mathbf{C}^{(0)}$ to be real. After the choice is made, we can solve the eigenvalue equation iteratively so that the solution of the $(n-1)$ st iteration step is the foundation of the $n$th iteration step. Ideally, the eigenvectors approach the ground-state orbitals with each step until the change between two successive iteration steps does not exceed a chosen threshold, and the HF iteration is converged. During the HF iteration, the core Hamiltonian $\hat{H}^{(\text {core) }}$ remains constant and only the SCF potential $\hat{V}^{(\mathrm{scf})}$ changes according to the eigenvectors $\mathbf{C}_{j}$. As we will see later, the HF method does not converge for every value of the complex coupling strength that will be introduced in the Sec. III.

\section{B. Connection between the modified Hartree-Fock method and dynamical systems}

In this section, we analyze the properties of the HF Hamiltonian when a complex factor $\lambda$ is introduced. Moreover, we compare the HF iteration with the iteration underlying the Mandelbrot set. To this end, we insert a complex factor $\lambda$ in front of the mean-field potential

$$
\mathbf{F}=\mathbf{H}^{(\text {core })}+\lambda \mathbf{V}^{(\text {scf })} .
$$

This causes the whole eigenvalue equation [Eq. (16)] to depend on the factor $\lambda$, which can be written as

$$
\sum_{\nu} F_{\mu \nu}^{(n)}\left(\mathbf{C}^{(n-1)}, \lambda\right) C_{\nu j}^{(n)}(\lambda)=\epsilon_{j}^{(n)}(\lambda) C_{\mu j}^{(n)}(\lambda) .
$$

If $\lambda$ is complex, the eigenvectors $C_{\nu, j}^{(n)}$ will become complex, too, and so will the orbital energies $\epsilon_{j}^{(n)}$. However, Hamiltonians with real $\lambda$ and, especially, the Hamiltonian with the physical electron-electron repulsion at $\lambda=1$ remain real.

Enforcing symmetry of the Fock matrix (Sec. II A) is the first important choice for our modified HF method. The second choice is the guess on the initial eigenvectors $\mathbf{C}_{j}^{(0)}$ to create the first Hamiltonian operator. We start every iteration with the eigenvectors of the Hamiltonian at $\lambda=0$. Thus, the initial Hamiltonian (zeroth step) does not include any electron-electron interaction

$$
F_{\mu \nu}^{(0)}=H_{\mu \nu}^{(\text {core })} \in \mathbb{R} .
$$

The resulting real eigenvectors $\mathbf{C}_{j}^{(0)}$ of $\mathbf{F}^{(0)}$ lead to hydrogenlike wave functions for nuclear charge $Z$. Subsequent iterations employ Eq. (17) for a given $\lambda$. By varying the parameter $\lambda$, we control the strength of the mean-field potential and, therefore, the electron-electron interaction.

Our third choice for the procedure of the modified HF method is the convergence criterion which is based on the $\mathrm{HF}$ energy $E_{\mathrm{HF}}$ (see Ref. 9). The HF energy for an arbitrary iteration step is given by

$$
E_{\mathrm{HF}}=2 \sum_{i=1}^{N / 2}\left(T_{i i}+V_{i i}^{(\mathrm{ne})}\right)+\sum_{i=1}^{N / 2} \sum_{i<j}^{N / 2}\left(2 J_{i j}-K_{i j}\right) .
$$

The indices $i$ and $j$ run over the number of occupied orbitals. The direct and exchange terms are given by

$$
\begin{aligned}
J_{i j} & =\sum_{\rho \sigma} C_{\rho i} C_{\sigma j} J_{\rho \sigma}, \\
K_{i j} & =\sum_{\rho \sigma} C_{\rho i} C_{\sigma j} K_{\rho \sigma} .
\end{aligned}
$$

The HF energy is a functional of the orbitals $\varphi_{i}$, which are written in the basis set $\chi_{\rho}$ with coefficients $C_{\rho i}$. Since the coefficients depend on $\lambda$ [see Eq. (18)], the HF energy depends on $\lambda$ as well. We define the decrease in the absolute value of the HF energy difference between the $n$th and $(n-1)$ st iteration step $\left(\Delta E_{\mathrm{HF}}^{(n)}\right)$ beneath a fixed threshold energy $\Delta E_{\mathrm{conv}}$ as the convergence criterion 


$$
\Delta E_{\mathrm{HF}}^{(n)} \equiv\left|E_{\mathrm{HF}}^{(n)}(\lambda)-E_{\mathrm{HF}}^{(n-1)}(\lambda)\right| \leq \Delta E_{\mathrm{conv}} .
$$

The convergence criterion for the energy assumes also the convergence of the orbitals. Here, recall that, motivated by the mathematical analysis of many-body perturbation theory, we restrict ourselves to the mathematical question of the HF convergence behavior and do not aim to optimize the HF method itself. This means that after initiating the orbitals, we run $\mathrm{HF}$ with the respective $\lambda$ and check for convergence with the criterion described above. This may have the consequence that especially for the physical value $\lambda=1$, convergence is not achieved, which is no problem for our further considerations.

Since the HF method is iterative, the orbitals can be considered to evolve in the course of the iteration. Therefore, the HF method can be understood as a discrete dynamical system, where each iteration step is interpreted as a time step. This holds also for the standard $\operatorname{HF}(\lambda=1)$ and does not depend on the complex nature of $\lambda$. Discrete dynamical systems are, e.g., the logistic map, ${ }^{31}$ the Sierpinski triangle (produced with the so-called chaos game ${ }^{32}$ ), the Cantor middlethird set, ${ }^{33}$ or the Mandelbrot set. ${ }^{34}$ In the following, we focus on the Mandelbrot set, which arises from an iteration process. The function $Q_{\lambda}^{(n)}$ of the Mandelbrot set is the family of quadratic functions

$$
Q_{\lambda}^{(n)}\left(z^{(n-1)}\right)=\left[z^{(n-1)}\right]^{2}+\lambda=z^{(n)},
$$

where $z$ and $\lambda$ are complex numbers and $n$ denotes the number of steps taken. As in the HF iteration, an initial $z^{(0)}$ must be provided, which in this case is zero. Therefore, $z^{(1)}=\lambda$.

The Mandelbrot set $M$ is defined by the set of $\lambda$ for which the absolute value of the sequence $Q^{(n)}$ remains finite for an infinite number of iteration steps

$$
M=\left\{\lambda \in \mathbb{C}\left|z^{(0)}(\lambda) \neq 0,\right| z^{(n)}(\lambda) \mid \dashv \infty\right\} .
$$

Thus, the Mandelbrot set is the subset of complex $\lambda$ for which the quadratic function defined in Eq. (24) gives rise to a nondivergent sequence. The Mandelbrot set allows several types of sequences for $z^{(n)}$ that can exist in the iteration process. One type of sequence is, for example, $z^{(1)}=z^{(2)}=\ldots=z^{(k)}$, and those points are called fixed points for a particular $\lambda$ because the value of $z^{(k)}$ is not changing during the iteration. Other types are the $k$-cycles, where a sequence repeats after $k$ iteration steps $Q_{\lambda}^{(k)}\left(z^{(l)}\right)=z^{(l)}$, where $k$ and $l$ are integers. Note here that iterations approaching a $k$-cycle do not fulfill the general definition for convergence since they do not approach a single value.

Since already a simple iteration like the Mandelbrot set produces a highly fragmented geometric figure, it is a legitimate question to ask whether a more complex iteration such as the HF iteration generates a complex fragmented geometric figure too. We use the fractal dimension to assign a value to the degree of fragmentation of a geometric figure. Because estimating the fractal dimension of unknown geometrical figures is an important subject in this work, we give in Sec. II C a method to obtain fractal dimensions. More precisely, it should be determined whether the SCF set exhibits a noninteger fractal dimension. While the Mandelbrot set iterates twodimensional numbers (one dimension for the real part of $z^{(n)}$ and one for its imaginary part), the HF iteration involves a set of complex eigenvectors. Furthermore, the convergence criterion of the $\mathrm{HF}$ iteration differs from the criterion of the Mandelbrot set. Since we do not know a priori the critical absolute value of the HF energy, we cannot apply a similar criterion as it is done in the Mandelbrot set. For instance, our chosen convergence criterion in Eq. (23) for the HF iteration does not allow $k$-cycles and asks only for fixed points, i.e., the converged HF energies. This is a stronger condition on the convergence and can lead to a smaller size of the SCF set. In contrast, the Mandelbrot set excludes only those $\lambda$ values that cause a divergence to infinity when iterating Eq. (24) but still allows $k$ cycles. In our case, $\lambda$ values leading to $k$-cycles will be considered diverged with regard to the SCF set. When applying our stronger HF convergence criterion to the Mandelbrot set in Eq. (24), the points that are responsible for the boundary of the Mandelbrot set are excluded and the edges of the set become smooth instead of fragmented. Hence, the convergence criterion can influence the value of the fractal dimension.

Both sets exhibit analogies in the way they are constructed and can be considered as discrete dynamical systems. Taking the similarities between the HF iteration and the Mandelbrot set as an inspiration, we investigate the fractal structure and the convergence behavior of the $\mathrm{HF}$ method.

\section{Calculating the fractal dimension}

In this section, we motivate the use of a fractal dimension and present a method to estimate such a dimension. As the name fractal dimension suggests, the dimension of a geometric figure need not be necessarily an integer but can also be a fraction. There are many ways to estimate a fractal dimension. 35,36 We decide to use the box-counting method ${ }^{36,37}$ since it finds usage in a wide variety of research fields, e.g., in characterizing textures in optical coherence tomography images, ${ }^{38,39}$ in estimating rock fracture surfaces, ${ }^{35}$ in archeology, ${ }^{40}$ or in the geometric characterization of the galaxy distribution. ${ }^{41}$ Here, the box-counting method itself provides the definition for the dimension of the geometric figure, such that the dimension estimated by the box-counting method is also referred to as the box-counting dimension. Still, we favor the term fractal dimension because of its common use for fractals. Because the fractal dimension can be a statement for the roughness or smoothness of a landscape or a coast-line, ${ }^{42,43}$ we, too, will take the following estimated fractal dimension as an indicator for the roughness and fragmentation of margins of the considered geometric objects. In contrast to the references mentioned above, our objects will be filled and, therefore, approach more a twodimensional plane than a one-dimensional line. This will result in values close to two. Hereby, we call a geometric figure with a fractal dimension of 1.7 more fragmented than a figure with a dimension of 1.9 , which is more similar to a twodimensional object with a smoother margin.

The starting point of the box-counting method is in our case always a geometric figure that consists of discrete points 
in the two-dimensional $\lambda$ space. In order to determine its fractal dimension, in the first step, the figure is covered with disjunct squares of side length $l$. Then, the number of squares is determined which contain at least one point of the geometric figure. The total number of squares that are necessary to cover the whole figure is denoted by $N(l)$ and depends obviously on $l$. The relation between the number $N(l)$, the side length $l$, and the fractal dimension $D_{F}$ is given by

$$
N(l)=\frac{c}{l^{D_{F}}} \text { as } l \rightarrow 0 .
$$

Here, $c$ is a real constant. Taking the base 10 logarithm of Eq. (26) gives

$$
\log N(l)=D_{F} \log \frac{1}{l}+\log c
$$

Therefore, plotting $\log N(l)$ as a function of $\log \frac{1}{l}$ yields a straight line with the fractal dimension as its slope.

\section{Normalization, symmetry properties, and implementation of the Hartree-Fock method}

In this section, the mathematical properties of the $\mathrm{HF}$ method and, in particular, the consequences of matrix symmetry will be examined.

As a consequence of the construction principles laid out in Secs. II A and II B, the HF Hamiltonian for a given $\lambda$ is a complex symmetric matrix $\left(\mathbf{F}^{\top}=\mathbf{F}\right)$ and not, in general, a Hermitian matrix $\left(\mathbf{F}^{\dagger} \neq \mathbf{F}\right)$. The eigenvectors $\mathbf{C}_{j}$ of the $\mathrm{HF}$ Hamiltonian may be assumed to be orthogonal to each other, in the sense that $\mathbf{C}_{i}^{\top} \mathbf{C}_{j}=0$ for $i \neq j .{ }^{44}$ In every iteration step, the eigenvectors are calculated and normalized with respect to the complex symmetric norm

$$
\left\|\mathbf{C}_{j}\right\|=\sqrt{\mathbf{C}_{j}^{\top} \mathbf{C}_{j}}
$$

Complex symmetric $W \times W$ matrices have $W$ eigenvalues and eigenvectors. However, in the case of degenerate eigenvalues, the eigenvectors are not necessarily linearly independent. ${ }^{44}$ Complex symmetric matrices find widespread use in scattering theory. ${ }^{44-46}$

In the following, we show that as a consequence of complex symmetry instead of hermiticity, there exists a simple connection between the eigenpairs associated with $\lambda$ and the eigenpairs associated with the complex conjugate of $\lambda$. We start with complex conjugating the eigenvalue equation of $\mathbf{C}_{j}(\lambda)$

$$
\left(\sum_{\nu} F_{\mu \nu}^{(n)}\left(\mathbf{C}^{(n-1)}, \lambda\right) C_{\nu j}^{(n)}\right)^{*}=\left(\epsilon_{j}^{(n)}(\lambda) C_{\mu j}^{(n)}(\lambda)\right)^{*} .
$$

Because of Eqs. (12), (13), and (17) and the assumption that $H_{\rho \sigma}^{\text {(core) }}$ and $(\rho \mu \mid \sigma \nu)$ are real, we can write

$$
\sum_{\nu} F_{\mu \nu}^{(n)}\left(\mathbf{C}^{(n-1) *}, \lambda^{*}\right)\left(C_{\nu j}^{(n)}(\lambda)\right)^{*}=\left(\epsilon_{j}^{(n)}(\lambda)\right)^{*}\left(C_{\mu j}^{(n)}(\lambda)\right)^{*} .
$$

On the other hand, the eigenvalue equation at $\lambda^{*}$ reads

$$
\sum_{\nu} F_{\mu \nu}^{(n)}\left(\mathbf{C}^{(n-1)}, \lambda^{*}\right) C_{\nu j}^{(n)}\left(\lambda^{*}\right)=\epsilon_{j}^{(n)}\left(\lambda^{*}\right) C_{\mu j}^{(n)}\left(\lambda^{*}\right) .
$$

Hence, we may conclude that

$$
\begin{gathered}
{\left[\mathbf{C}_{j}(\lambda)\right]^{*}=\mathbf{C}_{j}\left(\lambda^{*}\right),} \\
{\left[\epsilon_{j}(\lambda)\right]^{*}=\epsilon_{j}\left(\lambda^{*}\right) .}
\end{gathered}
$$

This means that if one iteration starts at the complex parameter $\lambda$ and another iteration starts at $\lambda^{*}$, the eigenvectors and eigenvalues of the two iterations are complex conjugates of each other. This, in turn, means that the SCF convergence behavior at $\lambda$ is the same as that at $\lambda^{*}$, so that the SCF set is symmetric with respect to the real $\lambda$ axis. Of course, this statement holds only if every iteration starts with the same initial vectors $\mathbf{C}_{j}^{(0)}$, which is ensured by Eq. (19).

The HF program used in this work is based on Refs. 47 and 48. For the calculations, we use a radial pseudospectral Gauß-Lobatto grid with a length of $r_{\max }=40 \mathrm{a}$.u. and $N_{\text {grid }}=200$ grid points whose distribution is concentrated near $r=0$. The mapping parameter $\zeta$ (Ref. 47) is set to 0.461 . Those grid parameters are chosen such as to ensure that they have no influence on the convergence behavior of the HF iteration.

\section{RESULTS AND DISCUSSION}

In this section, the HF method for the closed-shell atoms helium, neon, and argon is investigated. In particular, we study the effects of the factor $\lambda$ on the results of the HF method.

\section{A. Helium}

First, we present a general strategy to obtain the SCF set of helium and the box-counting method to calculate the fractal dimension of the SCF set. Then, the contour plots of the orbital energies for the SCF set will be analyzed, and the radial wave function of helium will be examined for different $\lambda$ values. Additionally, we investigate the convergence behavior of the HF method. The procedures for obtaining the SCF set, the contour plot, and the radial orbital wave functions also find use in the calculations for neon and argon in Secs. III B and III C, respectively.

\section{Strategy for obtaining the SCF set and its analysis}

We systematically run the HF method for different complex values of $\lambda$. To cover the complex plane, we use polar coordinates for $\lambda$

$$
\lambda=\rho \mathrm{e}^{i \vartheta},
$$

with a radial coordinate $\rho$ and an angular coordinate $\vartheta$. After one HF iteration is accomplished, the polar coordinates of $\lambda$ are varied by variation steps $\rho_{\text {step }}$ and $\vartheta_{\text {step }}$.

We check at every iteration step whether the $n$th and $(n-1)$ st HF energies satisfy the convergence criterion of Eq. (23) or not. In the following, we set the convergence threshold energy $\Delta E_{\text {conv }}$ to $10^{-10}$ a.u. If the convergence 
criterion is fulfilled, the iteration procedure aborts and the factor $\lambda$ is plotted in the complex coupling-strength plane. To have a better foundation for analyzing the data, we color the points according to the number of iteration steps $n_{\max }$ that are necessary to satisfy the convergence criterion. The iteration steps necessary for convergence are sorted in bins of 30 , and the associated $\lambda$ points are plotted in the corresponding color. The maximum number of iteration steps is set to 330 so that after 330 steps, the HF iteration aborts automatically without necessarily satisfying the convergence condition. $\lambda$ points for which the iteration does not fulfill the criterion within 330 steps are not plotted.

We apply this strategy to helium $(Z=2)$ and plot the resulting SCF set in Fig. 1. The scanning parameters for the radial and angular parts are $\rho_{\text {step }}=0.05$ and $\vartheta_{\text {step }}=0.003 \pi$ which are also used for the SCF sets of neon and argon in Secs. III B and III C. The SCF set $\Omega_{\mathrm{He}}$ splits into different areas if we classify the points by the number of iteration steps, $n_{\max }$, required for convergence.

For real values of $\lambda$, the electron-electron interaction varies from repulsion for positive $\lambda$ to attraction for negative $\lambda$. The repelling electron potential can be increased until $\operatorname{Re}(\lambda)=1.75$; beyond this value, the repulsion is too strong to allow SCF convergence, as shown in Fig. 1. In the opposite direction, when $\operatorname{Re}(\lambda)$ becomes negative, the electronelectron attraction facilitates the convergence of the HF method, leading to the asymmetric behavior with respect to the imaginary axis. The symmetry with respect to the real axis was already predicted in Sec. IID. One might assume that the HF method just needs more than 330 iteration steps to reach convergence for values of $\lambda$ outside the SCF set $\Omega_{\mathrm{He}}$. However, this assumption is not always correct, as we will see later (Fig. 7).

Next, we analyze several parts of the SCF set in more detail. For better visibility, we color those points black which are not converged within the convergence criterion. In Fig. $2(a)$, we refine the scanning parameters for $\lambda$. We start with a
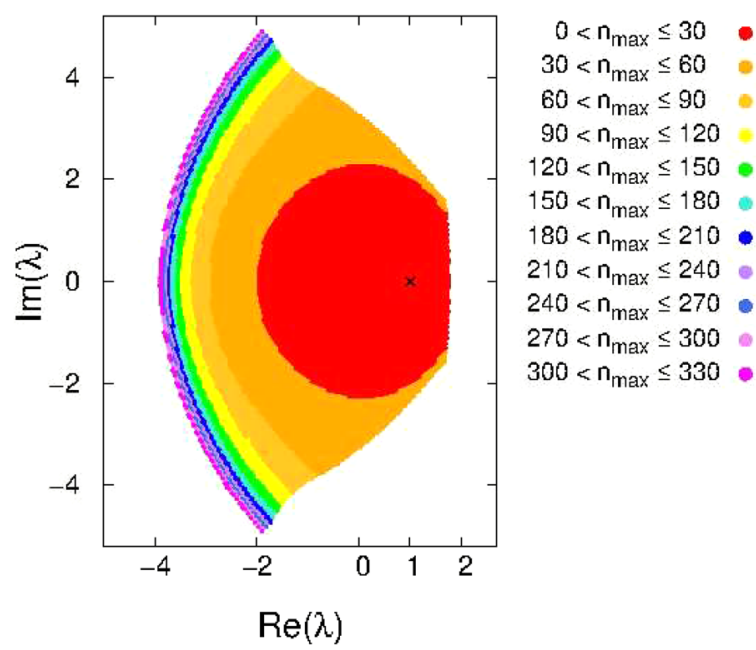

FIG. 1. The SCF set for helium in the complex coupling-strength plane: It is generated by plotting the parameter $\lambda$ for which the HF method converges. The colored areas represent the number of iteration steps necessary for reaching convergence $n_{\max }$. The physical value at $\lambda=1$ is marked by a black cross. radial part of $\rho_{\text {start }}=3$ and continue in steps of $\rho_{\text {step }}=0.005$ to $\rho_{\text {end }}=5.4$. The scanning parameters for the angular part are $\vartheta_{\text {start }}=0.595 \pi, \vartheta_{\text {end }}=0.745 \pi$, and $\vartheta_{\text {step }}=0.0003 \pi$. We recognize slightly fragmented borders that do not seem to be totally smooth. Besides, at this higher resolution, three spots appear in an area of converged SCF sequences where the HF method does not converge within 330 steps. Therefore, another refinement of the scanning parameters is performed to investigate those areas. The scanning parameters for Figs. 2(b) and 2(c) are $\rho_{\text {step }}=0.0005$ and $\vartheta_{\text {step }}=0.00005 \pi$.

In Fig. 2(b), the region of nonconvergent points is bigger than in (c), where the region is split into several small parts. The position of the diverging points in Fig. 2(c) is close to the border between the zone with $n_{\max } \leq 90$ and the zone with $90<n_{\max }$. In this area of diverged points, the borderline is more fragmented than along the remaining border. This observation implies that the diverged points are responsible for the increasing degree of fragmentation. However, the impact of the points on $n_{\max }$ of the surrounding convergent points cannot be greater than 30 iteration steps because in panel (b), we have a homogeneous region around the area of points, where we do not find any convergent points with an $n_{\max }$ higher than 90 or less than 61.

\section{Estimating the fractal dimension}

We apply the box-counting method in the following to estimate the fractal dimension of the SCF set $\Omega_{\mathrm{He}}$. First, we include all points plotted in Fig. 1. As derived in Eq. (27), the fractal dimension is the slope of a straight line when $\log (N)$ is plotted versus $\log (1 / l)$. The result is shown in Fig. 3. We obtain the slope by applying a damped leastsquare fit to the data points in the linear region. As we can observe, the graph loses its linear behavior for small and large box sizes $l$. For example, on the one hand, for squares with a large side length $l$, only a few boxes are necessary to cover the SCF set $\Omega_{\mathrm{He}}$. This has the effect that this slope generated with large $l$ cannot reflect the fragmentation of the margin of the set, and therefore, we exclude those values for the box-counting method. On the other hand, when $l$ becomes too small, the number of squares becomes equal to the number of data points. This is the case when the square size becomes smaller than our chosen scanning parameters. So, we have to restrict the box sizes to the chosen scanning parameters of the set. Nevertheless, we can identify in a certain region a slope on different scales, which is sufficient to estimate the fractal dimension. The question of the stability of computational algorithms and means for improvement of the box-counting method have been discussed in Refs. 49 and 50 .

We show the results for the fractal dimensions in Table I. The fractal dimension is calculated as described above for the SCF set $\Omega_{\mathrm{He}}$ and for its SCF subsets $\Omega_{\mathrm{He}, L}$. The SCF subsets $\Omega_{\mathrm{He}, L}$ are defined by the parameter $L$, which limits the number of iteration steps $n_{\max }$ that the HF method needs to reach convergence. This means that a $\lambda$ dependent HF iteration belongs to a SCF subset $\Omega_{\mathrm{He}, L}$ if the number of the last iteration step $n_{\max }$ is equal to or less than the limit $L$. The SCF set $\Omega_{\mathrm{He}, 330}$ is equal to $\Omega_{\mathrm{He}}$. The obtained fractal 

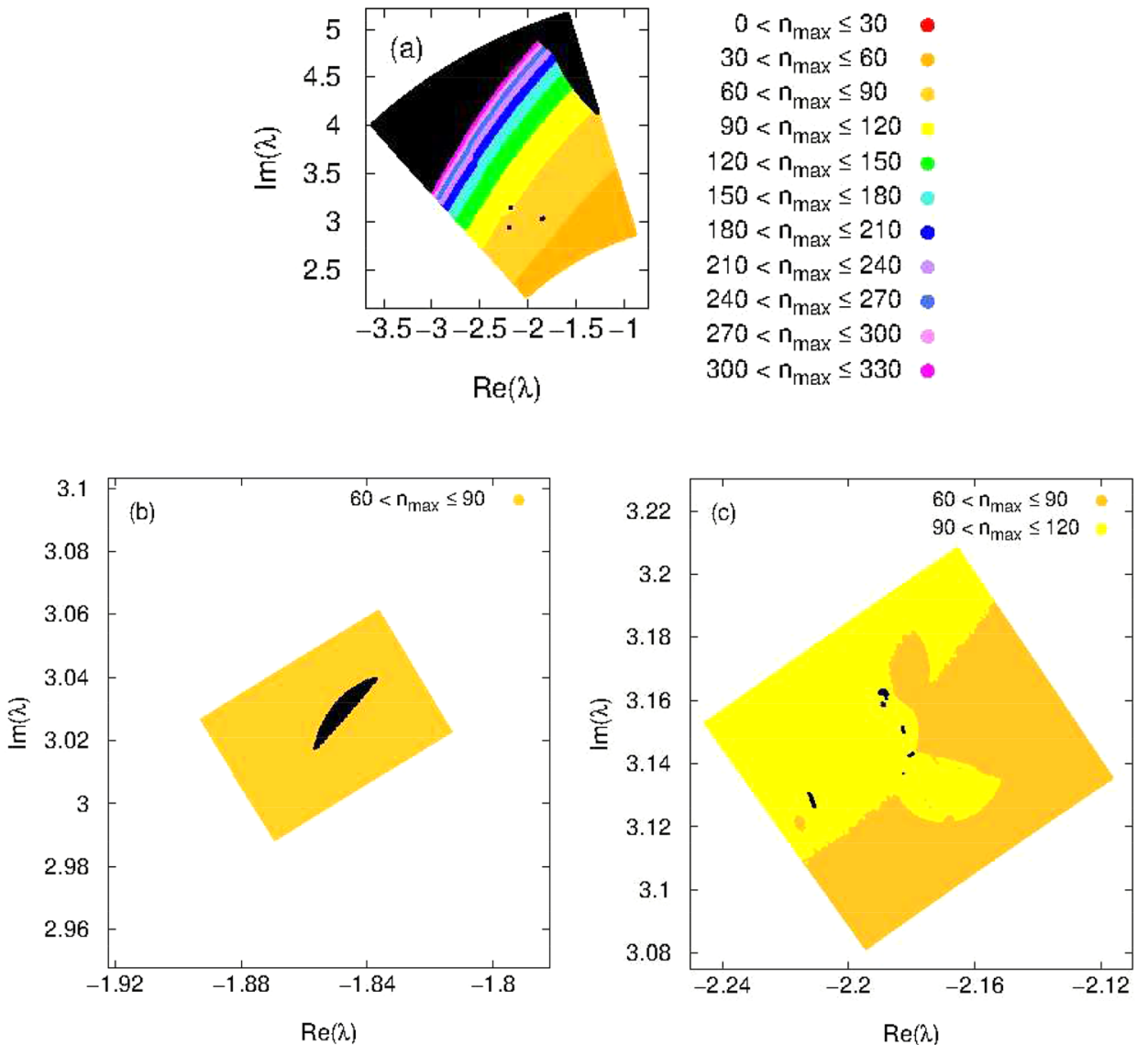

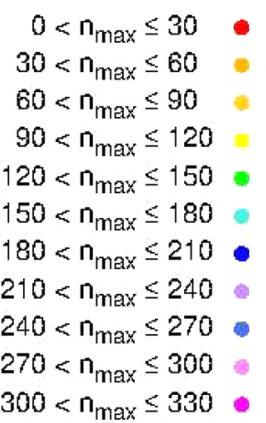

FIG. 2. Details of the upper left part of Fig. 1. All black-colored points represent $\lambda$ factors for which the HF method has not converged. Panels (b) and (c) provide close-up views of regions of divergence. Note that the scale in (b) is the same as in (c). dimensions for the subsets $\Omega_{\mathrm{He}, L}$ with $L=\{30,90,180\}$ are smaller than two within the statistical error. The results for the subsets $\Omega_{\mathrm{He}, L}$ with $L=\{270,330\}$ are slightly beneath two but include within the error of the value of two.

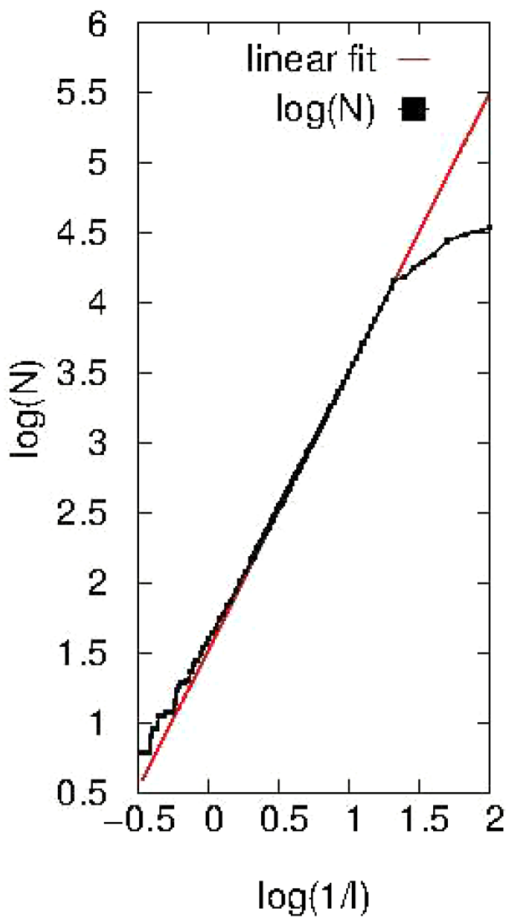

FIG. 3. Box-counting method for the SCF set of helium $\Omega_{\mathrm{He}}$. The number of disjunct squares $N$ that are necessary to cover $\Omega_{\mathrm{He}}$ is given as a function of the side length $l$.
An intuitive way to explain why the subsets have a fractal dimension less than two is that the subsets are slightly fragmented on their edges and, therefore, do not possess enough points to fill out a full two-dimensional figure. This explanation will become more evident, when taking a look at the fractal dimensions of neon and argon, where the edges of the SCF sets are far more fragmented, and the values of the fractal dimensions decrease. Since we obtain a non-integer fractal dimension, we give evidence that the SCF set, at least for small $L$, is a fractal. ${ }^{26}$ However, note that we have not proved self-similarity of the SCF set on different scales. In fact, even for the fractal structures in nature, self-similarity on different scales cannot always be shown. ${ }^{51,52}$

\section{Orbital properties of the SCF set and investigation of convergence behavior}

In this section, we investigate the orbital energies in the SCF set as a function of $\lambda$ and analyze the shape of the radial

TABLE I. Fractal dimension $D_{\mathrm{F}}$ of five subsets of the SCF set $\Omega_{\mathrm{He}, L}$, which consist of $\lambda$ points, for which the HF method is converged within a limit of $L$ iteration steps. Here, $n_{\max }$ is the number of the last iteration when convergence is reached.

\begin{tabular}{lcc}
\hline \hline Element & $\Omega_{\mathrm{He}, L}$ & $D_{\mathrm{F}}$ \\
\hline Helium & $n_{\max } \leq 30$ & $1.948 \pm 0.038$ \\
& $n_{\max } \leq 90$ & $1.966 \pm 0.005$ \\
& $n_{\max } \leq 180$ & $1.964 \pm 0.004$ \\
& $n_{\max } \leq 270$ & $1.992 \pm 0.041$ \\
& $n_{\max } \leq 330$ & $1.987 \pm 0.046$ \\
\hline
\end{tabular}



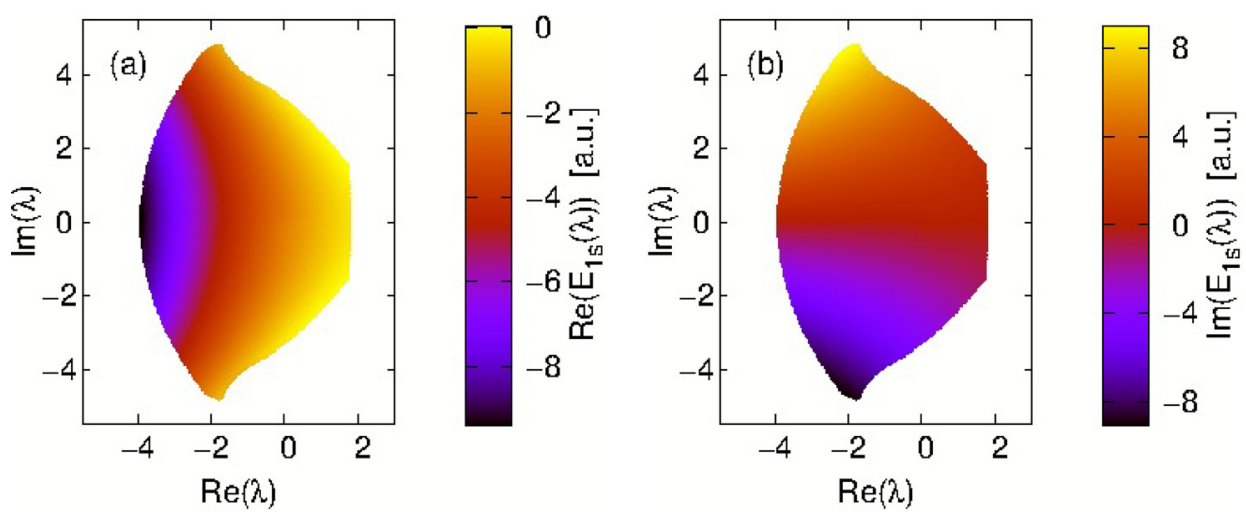

FIG. 4. Contour plot of the 1s orbital energy $E_{1 \mathrm{~s}}(\lambda)$ of helium in a.u. Panel (a) shows the real part and (b) the imaginary part of $E_{1 \mathrm{~s}}(\lambda)$. Only energies associated with converged $\mathrm{HF}$ iterations are taken into account.

wave function of the orbitals for various $\lambda$. At the end, we describe the convergence behavior of the HF method.

We plot in Fig. 4 the orbital energies in a contour plot in the complex coupling-strength plane. The SCF set is generated as described in Sec. III A 1. Here, we plot the converged orbital energy of the $1 \mathrm{~s}$ orbital $E_{1 \mathrm{~s}}(\lambda)$ of helium irrespective of $n_{\max }$. Consequently, the resulting figure has the same shape as in Fig. 1, but the points are colored according to the orbital energy to which the particular $\lambda$ leads. In Fig. 4(a), we plot the real part of the orbital energies and in (b) the imaginary part.

It is apparent that the orbital energies exhibit a symmetry with respect to the real axis. We derived this property already in Eq. (33), where we showed that the orbital energy at $\lambda$ is the complex conjugate of the orbital energy at $\lambda^{*}$. Thus, the real part of $E_{1 \mathrm{~s}}(\lambda)$ is symmetric, and the imaginary part is antisymmetric with respect to the real axis.

Moreover, the real part and the imaginary part of the orbital energy change smoothly when the electron-electron interaction, i.e., the factor $\lambda$, is varied, and the contour plot of the orbital energy does not reflect the fragmented shape of the SCF subsets $\Omega_{\mathrm{He}, L}$ (see Fig. 2). This smooth behavior of the orbital energy is not very surprising because we do not expect a jump in the energy of a converged HF iteration when we vary the factor $\lambda$ by an infinitesimal step. Thus, the contour plot looks like a continuous function. For the points that are not converged and therefore are not presented in Fig. 4, we cannot obtain an orbital energy so that the contour plots have only a continuous shape in the region of the SCF set.

In Fig. 5, we plot the energy of the 1s orbital of helium along the real $\lambda$ axis. In addition, we indicate in Fig. 5 the $1 \mathrm{~s}$

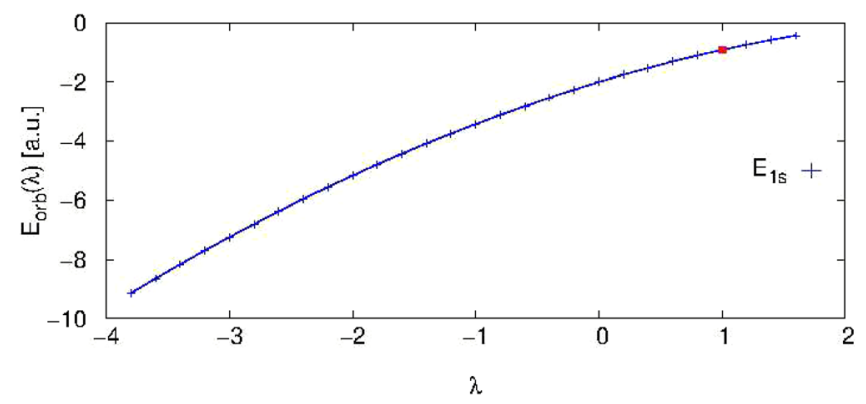

FIG. 5. Energy, in a.u., of the 1s orbital of helium along the real axis in the $\lambda$ plane. We take only those values of $\lambda$ into account which lead to convergence of the HF method. The red point indicates the HF orbital energy at $\lambda=1$ obtained with a standard HF code. orbital energy of helium at $\lambda=1$, computed using the standard Hartree-Fock procedure described in Ref. 47. In the plot, we recognize a continuous decrease in the orbital energy as $\lambda$ decreases and becomes negative. This reduction of the orbital energy is due to the decreasing electronelectron interaction, which switches from repulsion to attraction.

In Fig. 6, we plot, for four representative values of $\lambda$, the converged radial wave function $u_{\lambda, \text { orb }}(r)$ of the 1 s orbitals versus the distance from the nucleus $r$ in a.u. Here, the index "orb" represents the converged orbitals for a given parameter
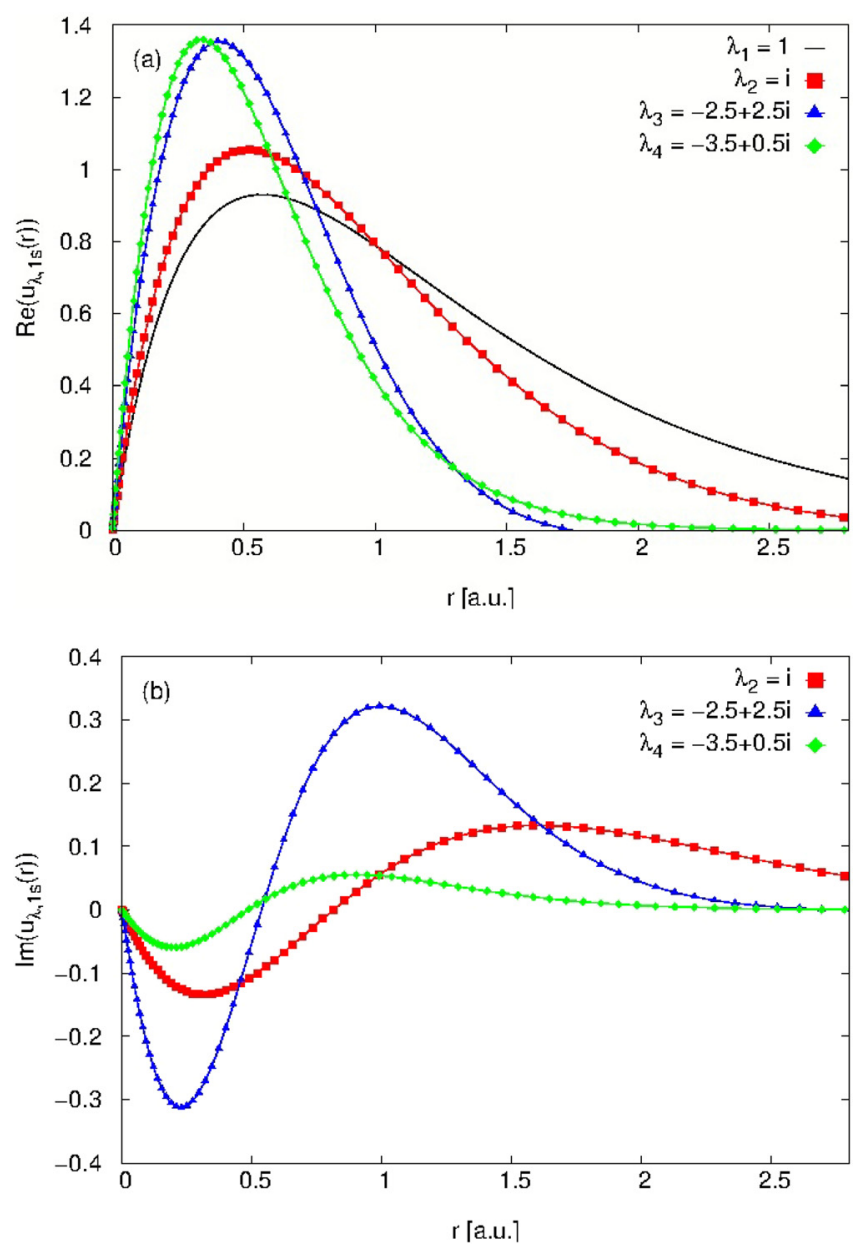

FIG. 6. Radial wave functions $u_{\lambda, 1 \mathrm{~s}}(r)$ of the $1 \mathrm{~s}$ orbital of helium for four different values of $\lambda$ after convergence of the HF method is reached. (a) Real part of $u_{\lambda, 1 \mathrm{~s}}(r)$ and (b) imaginary part. 
$\lambda$, which are related to the hydrogen-like orbitals at $\lambda=0$. Therefore, we use the standard quantum numbers of the hydrogen atom, the principal quantum number $n$, and the angular momentum quantum number $l$, for labelling the resulting orbitals. In the case of helium only, the 1s orbital is occupied, but, when discussing later, the case of neon "orb" also stands for the $2 \mathrm{~s}$ - and 2p-orbitals. Because $\lambda_{1}=1$ is real, the wave function $u_{\lambda_{1}, 1 \mathrm{~s}}(r)$ has no imaginary part and therefore does not appear in Fig. 6(b). As shown in Fig. 6(a), the maximum of the real part of $u_{\lambda_{1}, 1 \mathrm{~s}}(r)$ moves closer to the origin with decreasing $\operatorname{Re}(\lambda)$. This can be understood intuitively because it means that the orbitals for attracting electrons are smaller than orbitals in which the electrons repel each other. The imaginary part of $u_{\lambda, 1 \mathrm{~s}}(r)$ in Fig. 6(b) differs in its behavior and amplitude from the real part. Particularly, there is a clearly visible node below $r=1$. However, we observe also an analogy between $\operatorname{Re}\left[u_{\lambda, 1 \mathrm{~s}}(r)\right]$ and $\operatorname{Im}\left[u_{\lambda, 1 \mathrm{~s}}(r)\right]$ : For both sets of curves, the extrema move closer to the origin for smaller $\operatorname{Re}(\lambda)$. Furthermore, the magnitude of $\operatorname{Im}(\lambda)$ determines the amplitude of $\operatorname{Im}\left[u_{\lambda, 1 \mathrm{~s}}(r)\right]$ : The larger the value $\operatorname{Im}(\lambda)$, the larger the amplitude of $\operatorname{Im}\left[u_{\lambda, 1 \mathrm{~s}}(r)\right]$.

Now, we investigate the behavior of the HF method during the iteration process. To this end, we plot in Fig. 7 the absolute value of the HF energy difference $\Delta E_{\mathrm{HF}}^{(n)}$ between two successive iteration steps $n$ and $(n-1)$ versus the number of iteration steps $n$. The points have in each panel the same angular coordinate $\vartheta$ for $\lambda$ but different radial coordinates $\rho$. We choose the $\lambda$ points in such a way that in Fig. 7 (a), the points all lie on the positive real axis, in (b) on the positive imaginary axis, and in (c) on a line that starts from the origin and continues in the direction of $-1+\mathrm{i}$.

Figure 7(a) shows the evolution of $\Delta E_{\mathrm{HF}}^{(n)}$ during the iteration for five points on the positive real axis. We see that the convergence or divergence of the HF iteration depends strongly on the coupling-strength parameter $\lambda$. The HF energy for the points with a radial part of $0.5,1.7685$, and 1.7695 reaches the convergence threshold $\Delta E_{\text {conv }}$ within 50 iteration steps, while for parameters with radial parts of 1.769 and 6.5, no convergence is reached. For those points of $\lambda$, it seems that $\Delta E_{\mathrm{HF}}^{(n)}$ oscillates within a fixed energy interval and will never reach convergence. From this, we conclude that there exists no exact threshold along the line of the radial part $\rho$ where the HF iteration switches from only convergence to only divergence. Hence, the naive assumption raised in Sec. III A, which is with the increasing absolute value of $\lambda$, convergence will be achieved by merely increasing the number of iteration steps, is not correct. We find the same property of a missing clear border between convergent and divergent points also in Fig. 7(b) for parameters of $\lambda$ along the positive imaginary axis. Here, the HF iteration converges for $\lambda=3.269 \mathrm{i}$ but not for $\lambda=3.2685 \mathrm{i}$. The reason why we cannot see this intricate border in Fig. 1 is that the scanning parameters are too rough so that the substructure at the border is not resolved.

In Fig. 7(c), we show an effect connected to the chosen convergence criterion [see Eq. (23)]. In the figure, we see for $\rho=4.3$ and 4.5 a slow approach of $\Delta E_{\mathrm{HF}}^{(n)}$ to the convergence limit $\Delta E_{\text {conv }}$, but only the HF iteration at $\lambda=-3.04+3.04 \mathrm{i}$ fulfills the convergence criterion in less than or equal to 330 iteration steps. Moreover, the $\mathrm{HF}$ iteration at $\lambda=-3.18+$ $3.18 \mathrm{i}$ requires more steps but would fulfill the convergence criterion if the limit of the iteration steps were larger than 330 . This can be verified by running the HF method without limitation on the number of steps. For the HF iteration at $\lambda=-3.32+3.32 \mathrm{i}$, we observe again an oscillating $\Delta E_{\mathrm{HF}}^{(n)}$ that does not seem to converge even after an infinite number of iteration steps. We find this oscillatory behavior also for divergent $\lambda$ points that lie within the SCF set and are colored black in Fig. 2.

\section{B. Neon}

\section{SCF set and fractal dimension}

Following the same procedure as described in Sec. III A 1, the SCF set of neon $(Z=10)$ in the complex coupling-strength plane is obtained (Fig. 8). Additionally, we set the maximum $|\lambda|$ to $\rho_{\text {end }}$ and the maximum number of iteration steps for convergence again to 330 such that $\Omega_{\mathrm{Ne}}=\Omega_{\mathrm{Ne}, 330}$. The shape of the SCF set for neon differs in many aspects from the shape of the set $\Omega_{\mathrm{He}}$. The SCF set for neon in the left half-plane extends to $\rho_{\text {end }}=10$ and is therefore much bigger than the SCF set for helium. Additionally, the SCF set for neon exhibits two wings along the imaginary axis for $|\operatorname{Im}(\lambda)| \geqslant 4$. Those two wings contribute a significant part to the total SCF set. Apart from the two wings, we observe that the subsets are not connected anymore but show small substructures at their margins. Those substructures are big compared to those for helium, where we have to refine the resolution to observe the substructures. Moreover, the areas with a number of iteration steps $n_{\max }$ above 60 are small compared to those areas with $n_{\max }$ beneath 60 (red and orange colored points). Since the size of the SCF set is limited to the chosen maximal value for $\rho_{\text {end }}$, we can state that the SCF set $\Omega_{\mathrm{Ne}}$ would broaden with larger $\rho_{\text {end }}$. Therefore, we expect that also the areas with $60 \leq n_{\max }$ would extend with increasing $\rho_{\text {end }}$. Furthermore, we recognize an intricate structure at the borders between differently colored areas. While for helium, the fragmented structure was not very prominent for the chosen scanning parameters, for neon, the emerging substructure is striking, in particular in the outer regions where many different convergence areas mix.

The results for the fractal dimensions of the subsets of neon are shown in Table II. The SCF subsets $\Omega_{\mathrm{Ne}, 180}, \Omega_{\mathrm{Ne}, 270}$, and $\Omega_{\mathrm{Ne}, 330}$ have nearly the same amount of data points. Consequently, the associated margins display a similar degree of fragmentation, which leads to similar fractal dimensions for these SCF subsets. The dimensions are clearly smaller than two. These subsets have, like the subset $\Omega_{\mathrm{Ne}, 90}$, a small but discernible degree of fragmentation. For the SCF subset $\Omega_{\mathrm{Ne}, 30}$,

TABLE II. Fractal dimensions $D_{\mathrm{F}}$ of the SCF set of neon. The ensemble of points is divided into SCF subsets $\Omega_{\mathrm{Ne}, L}$ according to $n_{\max }$, the number of iterations necessary to bring the HF method to convergence for a given $\lambda$.

\begin{tabular}{lcc}
\hline \hline Element & $\Omega_{\mathrm{Ne}, L}$ & $D_{\mathrm{F}}$ \\
\hline Neon & $n_{\max } \leq 30$ & $1.855 \pm 0.032$ \\
& $n_{\max } \leq 90$ & $1.964 \pm 0.015$ \\
& $n_{\max } \leq 180$ & $1.963 \pm 0.009$ \\
& $n_{\max } \leq 270$ & $1.962 \pm 0.008$ \\
& $n_{\max } \leq 330$ & $1.962 \pm 0.008$ \\
\hline
\end{tabular}



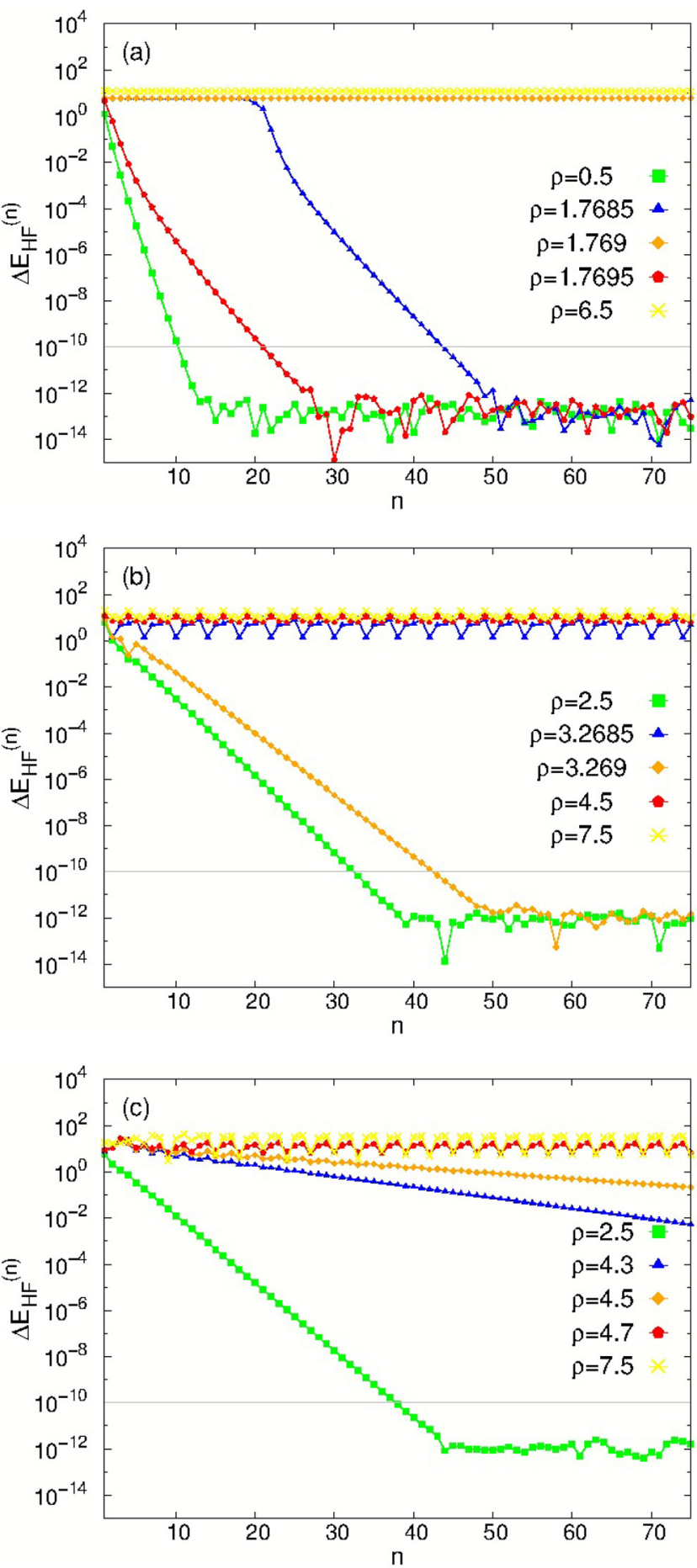

FIG. 7. Evolution of the energy difference $\Delta E_{\mathrm{HF}}^{(n)}$ as a function of the number of iteration steps $n$ for specific points of $\lambda=\rho \exp (\mathrm{i} \vartheta)$. In each panel, the angular coordinate $\vartheta$ is fixed: (a) $\vartheta=0$, (b) $\vartheta=\pi / 2$, and (c) $\vartheta=3 \pi / 4$. Note the logarithmic scale of the energy axis. The gray line indicates the threshold energy $\Delta E_{\text {conv }}=10^{-10}$ a.u.

we obtain a fractal dimension of $D_{\mathrm{F}}=1.855 \pm 0.032$, which indicates a strongly fragmented structure. Although we did not carry out a search for self-similarity, the calculated fractal dimension well below two indicates a strong fragmentation.

\section{Orbitals of the SCF set}

In Fig. 9, we show the contour plots of the occupied orbital energies $1 s, 2 s$, and $2 p$ in the SCF set. Similar observations as

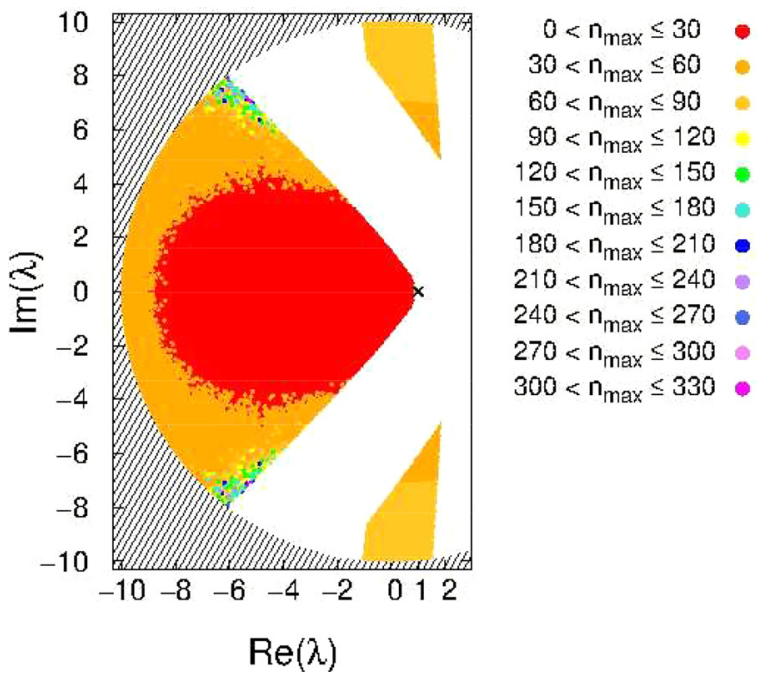

FIG. 8. SCF set of neon in the complex coupling-strength plane. The points are colored according to the number of iteration steps $n_{\max }$ that were necessary to bring the HF iteration to convergence. The hatched area indicates those $\lambda$ values that are not investigated for convergence. The physical value at $\lambda=1$ is marked by a black cross.

for helium can be made: The real parts are all symmetric about the real axis and the imaginary parts are antisymmetric. Furthermore, we recognize again a smooth change in the orbital energies with varying $\lambda$. As expected, the magnitudes of the $1 \mathrm{~s}$ orbital energies of neon are much larger than those of helium due to a tighter binding. One remarkable observation is that for the wings for $\lambda$ with $|\operatorname{Im}(\lambda)| \gtrsim 4$, all orbital energies are very close to zero for the real and imaginary parts.

In Fig. 10, we take a closer look at the orbital energies along the real axis of the contour plot. For this purpose, we vary convergent $\lambda$ from 0.95 to -10 , which corresponds to a range of $\lambda$ on the real axis. Additionally, we magnify the area around $\lambda=1$ to distinguish the graphs at their end points. The HF iteration at $\lambda=1$ does not reach convergence. This is because at every $\lambda$, we initiate the iteration using the $\lambda=0$ orbitals. Nevertheless, by extrapolating the curves of the orbital energies to $\lambda=1$, one can see that the resulting energies are consistent with standard HF.

When varying $\lambda$ from 0.95 to zero, we observe that the $2 \mathrm{~s}$ orbital energy is always lower than the energy of the $2 p$ orbital. At $\lambda=0$, the energies of the $2 \mathrm{~s}$ and $2 \mathrm{p}$ orbitals are equal. This is explained by the fact that for a vanishing SCF potential $(\lambda=0)$, the HF Hamiltonian equals the bare core Hamiltonian. Only if there is an interaction among the electrons, the degeneracy is lifted and the orbital energies split according to the angular momentum. Furthermore, as soon as $\lambda$ falls below zero, the energy of the $2 p$ orbital is smaller than the energy of the $2 \mathrm{~s}$ orbital. The crossing of the two curves can be seen in the inset of Fig. 10. In other words, for mutually attracting electrons, a $2 p$ electron has a higher binding energy than a 2 s electron.

No qualitatively new features arise in the radial orbital wave function, which are, therefore, not shown.

\section{Argon}

In Fig. 11, we show the SCF set $\Omega_{\mathrm{Ar}}$ of $\operatorname{argon}(Z=18)$, for which the same scanning parameters as for neon and 

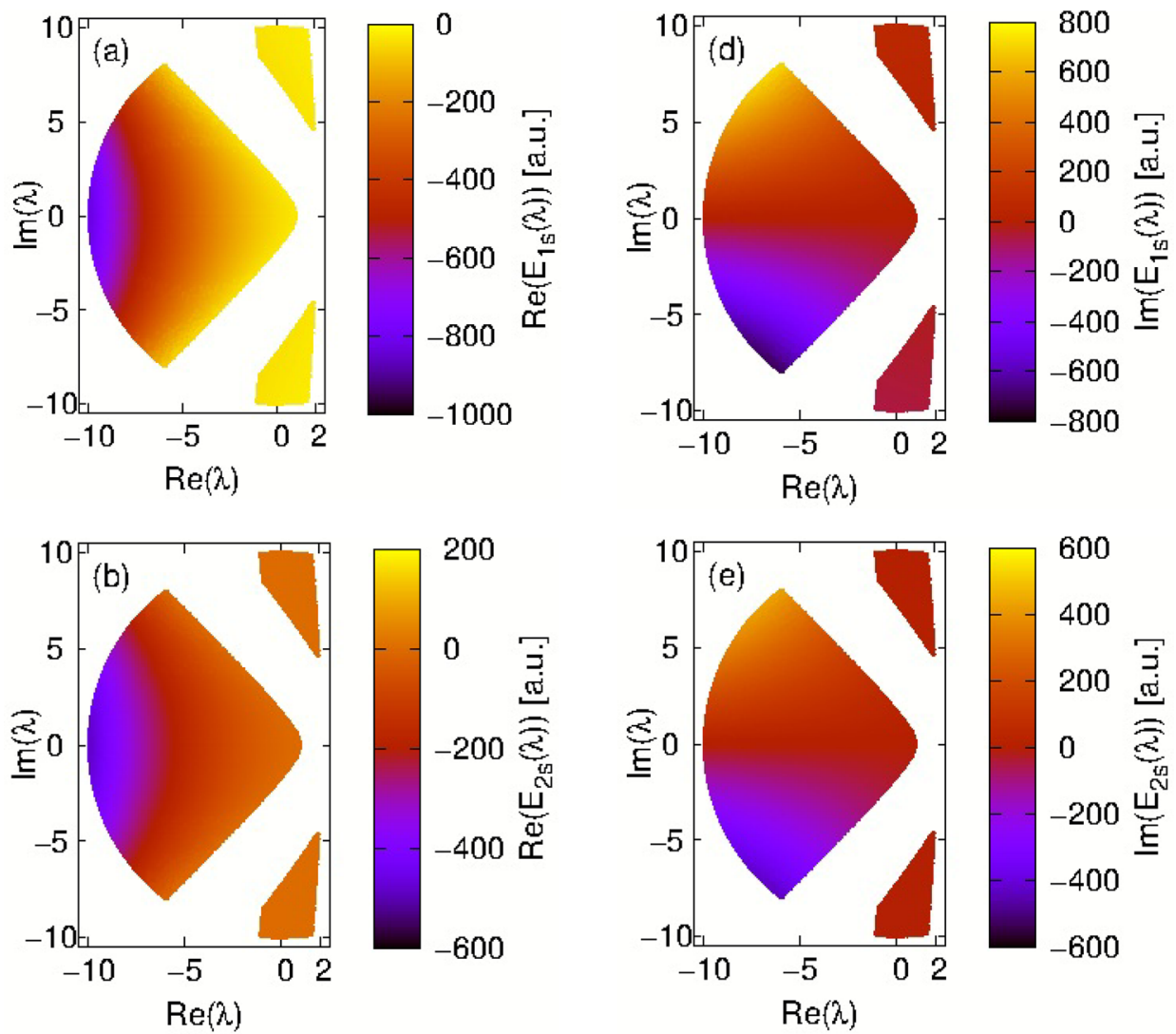

FIG. 9. Contour plots of the orbital energies $E_{\text {orb }}(\lambda)$ of neon in the complex coupling-strength plane. The real parts of the orbital energies of the $1 \mathrm{~s}$, $2 \mathrm{~s}$, and $2 \mathrm{p}$ orbitals are shown in panels (a)-(c), respectively, while their imaginary parts are presented in panels (d)-(f).
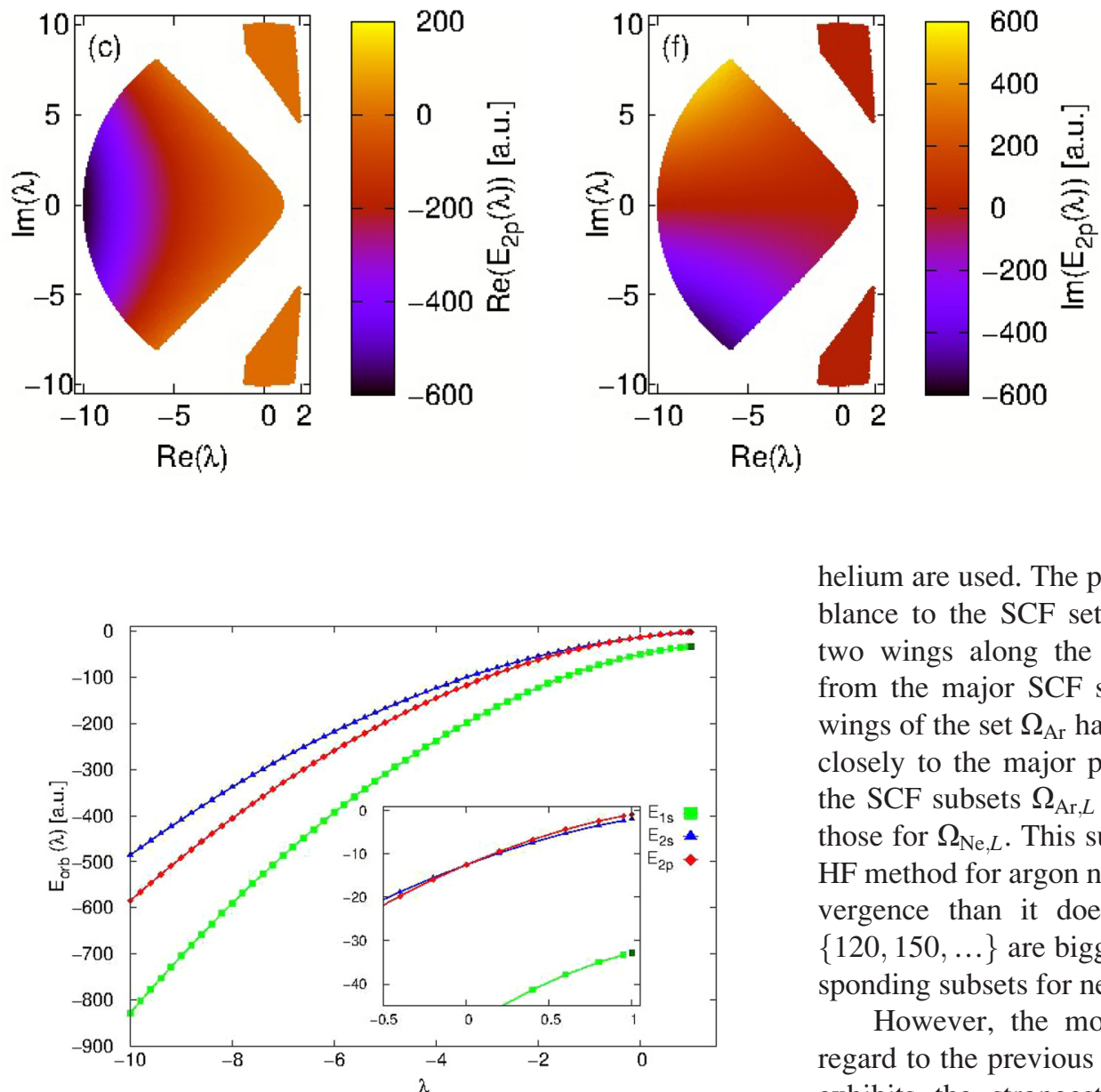

FIG. 10. Energies of the $1 \mathrm{~s}, 2 \mathrm{~s}$, and $2 \mathrm{p}$ orbitals of neon along the real axis for convergent $\lambda$. The points at $\lambda=1$ were calculated with standard HF.

helium are used. The pattern of the SCF set $\Omega_{\mathrm{Ar}}$ bears resemblance to the SCF set $\Omega_{\mathrm{Ne}}$. For instance, both sets exhibit two wings along the imaginary axis which are separated from the major SCF set. However, in contrast to $\Omega_{\mathrm{Ne}}$, the wings of the set $\Omega_{\mathrm{Ar}}$ have a smaller size and are located more closely to the major part of the set. Moreover, the sizes of the SCF subsets $\Omega_{\operatorname{Ar} . L}$ for $L=\{30,60,90\}$ are smaller than those for $\Omega_{\mathrm{Ne}, L}$. This suggests that for the same $\lambda$ values, the HF method for argon needs more iteration steps to reach convergence than it does for neon. SCF subsets with $L=$ $\{120,150, \ldots\}$ are bigger in the case of argon than the corresponding subsets for neon.

However, the most interesting property of $\Omega_{\mathrm{Ar}}$, with regard to the previous SCF sets $\Omega_{\mathrm{He}}$ and $\Omega_{\mathrm{Ne}}$, is that this set exhibits the strongest fragmentation, such that with our smallest chosen resolution, all of the subsets $\Omega_{\mathrm{Ar}, L}$ form visible substructures at their margins that seem to grow with 


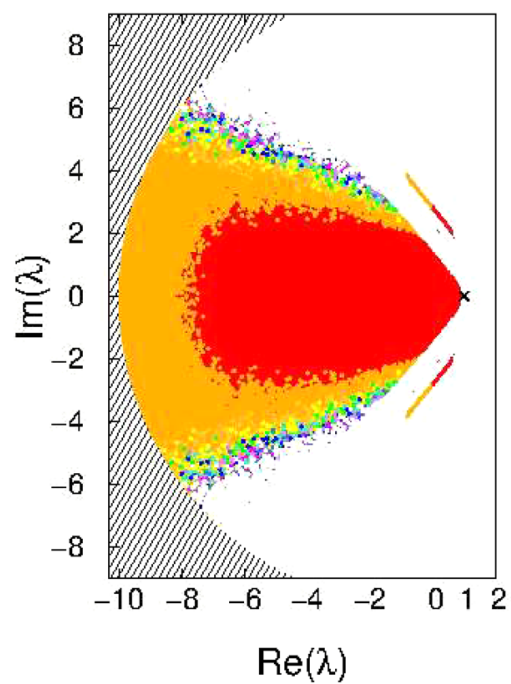

$0<\mathrm{n}_{\max } \leq 30$. $30<\mathrm{n}_{\max } \leq 60$. $60<\mathrm{n}_{\max } \leq 90$ $90<\mathrm{n}_{\max } \leq 120$ $120<n_{\max } \leq 150$ $150<\mathrm{n}_{\max } \leq 180$. $180<\mathrm{n}_{\max } \leq 210$. $210<\mathrm{n}_{\max } \leq 240$ $240<\mathrm{n}_{\max } \leq 270$. $270<\mathrm{n}_{\max } \leq 300$. $300<\mathrm{n}_{\max } \leq 330$

FIG. 11. SCF set of argon in the complex coupling-strength plane. The colored points represent the magnitude of the number of iteration steps $n_{\max }$ that were necessary to bring the HF iteration to convergence. The hatched area indicates those $\lambda$ values that are not investigated for convergence. The physical value at $\lambda=1$ is marked by a black cross.

decreasing $\operatorname{Re}(\lambda)$. Those substructures cause a high degree of fragmentation at the margins, which leads to correspondingly small fractal dimensions.

The fractal dimensions for the SCF set $\Omega_{\mathrm{Ar}}$ and its subsets $\Omega_{\mathrm{Ar} . L}$ are shown in Table III. The SCF subsets $\Omega_{\mathrm{Ar} . L}$ with $L=\{180,270,330\}$ have nearly the same fractal dimension, which is again due to the small difference in the number of points in those subsets and the similarly structured margins of those subsets. The SCF subset $\Omega_{\mathrm{Ar}, 30}$ exhibits clearly more fragmentation than $\Omega_{\mathrm{He}, 30}$ and $\Omega_{\mathrm{Ne}, 30}$. The values of the fractal dimensions are clearly beneath the value of two and confirm our observation in Fig. 11 that the SCF set $\Omega_{\text {Ar }}$ has the strongest fragmentation compared to $\Omega_{\mathrm{He}}$ and $\Omega_{\mathrm{Ne}}$. The subset $\Omega_{\mathrm{Ar}, 30}$ has the smallest fractal dimension, followed by $\Omega_{\mathrm{Ne}, 30}$ and then $\Omega_{\mathrm{He}, 30}$.

\section{CONCLUSIONS}

In this paper, we treated the Hartree-Fock iterative procedure as a dynamical system controlled by a complex coupling strength $\lambda$. Three particular choices on how to do this were made. First, complex-symmetric matrices were chosen. Second, every HF iteration was started without a mean-field potential $\hat{V}^{\text {(scf) }}$ so that the first eigenvectors correspond to hydrogen-like wave functions. Furthermore, we chose a criterion for the convergence of the HF iteration: The $\lambda$-dependent

TABLE III. Fractal dimensions $D_{\mathrm{F}}$ of the SCF set $\Omega_{\mathrm{Ar}}$ and of its SCF subsets $\Omega_{\mathrm{Ar}, L}$, with $L=\{30,90,180,270,330\}$.

\begin{tabular}{lcc}
\hline \hline Element & $\Omega_{\mathrm{Ar}, L}$ & $D_{\mathrm{F}}$ \\
\hline Argon & $n_{\max } \leq 30$ & $1.780 \pm 0.025$ \\
& $n_{\max } \leq 90$ & $1.887 \pm 0.045$ \\
& $n_{\max } \leq 180$ & $1.873 \pm 0.008$ \\
& $n_{\max } \leq 270$ & $1.872 \pm 0.009$ \\
& $n_{\max } \leq 330$ & $1.881 \pm 0.008$ \\
\hline \hline
\end{tabular}

HF iteration is considered to be converged when the absolute difference between two HF energies $\Delta E_{\mathrm{HF}}^{(n)}$ of two successive iteration steps is less than a critical value. In addition, a cutoff for the number of iteration steps was introduced.

Because the HF iteration can be considered as a discrete dynamical system, we compared the HF method with the iteration of the Mandelbrot set and discussed whether the SCF set should have a fractal dimension, like the Mandelbrot set. We came to the conclusion that, due to different notions of convergence, a fractal dimension for the SCF set of the HF iteration in the sense of the Mandelbrot set cannot be expected a priori.

Nevertheless, using the box-counting method, we found fractal dimensions for the atoms helium, neon, and argon. While for the SCF set of helium and its subsets there seems to be a small degree of fragmentation, with fractal dimensions slightly beneath two, we observe a stronger fragmentation for neon, where in particular, the subset $\Omega_{\mathrm{Ne}, 30}$ has the highest degree of fragmentation. However, we found that the SCF set of argon exhibits the strongest fragmentation, where the subset $\Omega_{\mathrm{Ar}, 30}$ has the smallest measured fractal dimension. In other words, with the increasing number of electrons, i.e., a more complex many-body problem, a smaller fractal dimension is measured.

We made the observation that the orbital energies change smoothly with $\lambda$ and that the contour plots exhibit a certain symmetry with respect to the real axis. In addition, we recognized a connection between the contraction of the radial orbitals and the decrease in $\operatorname{Re}(\lambda)$.

Furthermore, we observed two different types of behaviors of the HF method during the iteration process: In the first case, $\Delta E_{\mathrm{HF}}^{(n)}$ decreased with every iteration step until it fell beneath the threshold energy. In the second case, $\Delta E_{\mathrm{HF}}^{(n)}$ never reached the threshold energy and oscillated within a fixed energy interval during the iteration.

These results can serve as basis for further investigations of the convergence behavior of the HF method. Particularly, it will be interesting to study the implication for many-body perturbation theory. Here, we have chosen complex symmetry for the Fock matrix $\boldsymbol{F}$ [Eq. (16)] and thus complex orthogonality for the eigenvector matrix $\boldsymbol{C}$. It will be interesting to study the effects that complex conjugation of $C_{\mu i}$ in Eqs. (12) and (13) would have on the SCF convergence behavior in the complex $\lambda$ plane and also the influence of the initial guess for $\boldsymbol{C}$. Our analysis in the complex coupling-strength plane already indicates that fragmentation and fractal dimensions arise for the HF iteration. This leads to the question of the connection between the number of occupied orbitals and the corresponding fractal dimension and to the question how the spatial symmetries of the system are reflected in the fractal dimension of the respective HF set. Furthermore, the role of the strict convergence criterion in contrast to the criterion for the Mandelbrot set, which also allows for $k$-cycles, should be further investigated. Also, the question of self-similarity of the SCF sets presented in this work remains a topic for future studies. Finally, other two-body interactions, for example, nucleon-nucleon interactions for nuclei, could be considered in future studies. 


\section{ACKNOWLEDGMENTS}

We would like thank Otfried Geffert, Dietrich Krebs, and Stefan Pabst for help with the software modifications required for this project. Furthermore, we would like to thank Caroline Arnold for constructive comments on our manuscript. A.K. was supported through the Louise-Johnson fellowship by the excellence cluster "The Hamburg Centre for Ultrafast Imaging” under Grant No. DFG EXC 1074.

${ }^{1}$ R. S. Mulliken, Phys. Rev. 41, 49 (1932).

${ }^{2}$ N. Bohr, Nature 121, 580 (1928).

${ }^{3}$ F. Hund, Z. Phys. 36, 657 (1926).

${ }^{4}$ V. Fock, Z. Phys. 61, 126 (1930).

${ }^{5}$ J. C. Slater, Phys. Rev. 81, 385 (1951).

${ }^{6}$ P. Lykos and G. W. Pratt, Rev. Mod. Phys. 35, 496 (1963).

${ }^{7}$ E. J. Baerends, D. E. Ellis, and P. Ros, Chem. Phys. 2, 41 (1973).

${ }^{8}$ J. C. Slater, The Self-Consistent Field for Molecules and Solids: Quantum

Theory of Molecules and Solids (McGraw-Hill, New York, 1974), Vol. 4.

${ }^{9}$ A. Szabo and N. S. Ostlund, Modern Quantum Chemistry: Introduction to Advanced Electronic Structure Theory (Dover Publications, New York, Mineola, 1996)

${ }^{10} \mathrm{C}$. J. Cramer, Essentials of Computational Chemistry: Theories and Models (Wiley, Chichester, 2004).

${ }^{11}$ P. E. Cade and W. M. Huo, J. Chem. Phys. 47, 614 (1967).

${ }^{12}$ M. Rotenberg, Phys. Rev. 100, 439 (1955).

${ }^{13}$ K. T. R. Davies, S. J. Krieger, and M. Baranger, Nucl. Phys. 84, 545 (1966).

${ }^{14}$ A. Rosén, D. E. Ellis, H. Adachi, and F. W. Averill, J. Chem. Phys. 65, 3629 (1976).

${ }^{15}$ K. C. Kulander, Phys. Rev. A 36, 2726 (1987).

${ }^{16}$ S. Hirata, M. Head-Gordon, and R. J. Bartlett, J. Chem. Phys. 111, 10774 (1999).

${ }^{17}$ C. Møller and M. S. Plesset, Phys. Rev. 46, 618 (1934).

${ }^{18}$ F. H. Stillinger, J. Chem. Phys. 112, 9711 (2000).

${ }^{19}$ A. V. Sergeev and D. Z. Goodson, J. Chem. Phys. 123, 064105 (2005).

${ }^{20}$ C. G. Broyden, Math. Comput. 19, 577 (1965).

${ }^{21}$ D. D. Johnson, Phys. Rev. B 38, 12807 (1988).

${ }^{22}$ A. Baran, A. Bulgac, M. M. Forbes, G. Hagen, W. Nazarewicz, N. Schunck, and M. V. Stoitsov, Phys. Rev. C 78, 014318 (2008).

${ }^{23}$ R. L. Devaney, A First Course in Chaotic Dynamical Systems: Theory and Experiments (Westview Press, 2004).
${ }^{24}$ G. D. Birkhoff, Dynamical Systems (Colloquium Publications, American Mathematical Society, Rhode Island, 1927), Vol. 9.

${ }^{25}$ R. M. May, Nature 261, 459 (1976).

${ }^{26}$ B. B. Mandelbrot, The Fractal Geometry of Nature (Macmillan, New York, 1982)

${ }^{27}$ N. M. Hugenholtz, Phys. Rev. 96, 1158 (1954).

${ }^{28}$ C. G. Gray, G. Karl, and V. A. Novikov, Ann. Phys. 251, 1 (1996).

${ }^{29}$ C. C. J. Roothaan, Rev. Mod. Phys. 23, 69 (1951).

${ }^{30}$ N. Moiseyev, Non-Hermitian Quantum Mechanics, 1st ed. (Cambridge University Press, 2011).

${ }^{31}$ L. Kocarev and G. Jakimoski, Phys. Lett. A 289, 199 (2001).

${ }^{32}$ H. J. Jeffrey, Nucl. Acids Res. 18, 2163 (1990).

${ }^{33}$ N. C. B. Majumder, Am. Math. Mon. 72, 725 (1965).

${ }^{34}$ A. Douady, "Julia sets and the Mandelbrot set," in The Beauty of Fractals (Springer, Berlin, 1986).

${ }^{35}$ S. M. Miller, P. C. McWilliams, and J. C. Kerkering, "Ambiguities in estimating fractal dimensions of rock fracture surfaces," in Proceedings 31st US Symposium on Rock Mechanics, Balkema, The Netherlands, 1990.

${ }^{36} \mathrm{R}$. Engelking, Dimension Theory (North-Holland Publishing Company, New York, 1978).

${ }^{37}$ K. Foroutan-pour, P. Dutilleul, and D. L. Smith, Appl. Math. Comput. 105, 195 (1999).

${ }^{38}$ K. Falconer, "Box-counting dimension," in Fractal Geometry: Mathematical Foundations and Applications, 3rd ed. (Wiley, 2014).

${ }^{39}$ E. A. D. P. Popescu, Biomed. Opt. Express 1(1), 268 (2010).

${ }^{40}$ A. I. Penn and M. H. Loew, IEEE Trans. Med. Imaging 16(6), 930 (1997).

${ }^{41}$ C. T. Brown, W. R. T. Witschey, and L. S. Liebovitch, J. Archaeol. Method Theory 12(1), 37 (2005).

${ }^{42}$ R. Murdzek, Rom. J. Phys. 52(1/2), 149 (2007).

${ }^{43}$ C. M. Hagerhall, T. Purcell, and R. Taylor, J. Environ. Psychol. 24, 247 (2004).

${ }^{44}$ B. B. Mandelbrot, Science 156(3775), 636 (1967).

${ }^{45}$ R. Santra and L. S. Cederbaum, Phys. Rep. 368, 1 (2002).

${ }^{46}$ N. Moiseyev, Phys. Rep. 302, 83 (1997).

${ }^{47}$ U. V. Riss and H.-D. Meyer, J. Phys. B 26, 4503 (1993).

${ }^{48}$ L. Greenman, P. J. Ho, S. Pabst, E. Kamarchik, D. A. Mazziotti, and R. Santra, Phys. Rev. A 82, 023406 (2010).

${ }^{49}$ S. Pabst, L. Greenman, and R. Santra, XCID program package for multichannel ionization dynamics, Revision 402, 2011.

${ }^{50}$ A. Cetera, Chaos, Solitons Fractals 12, 475 (2001).

${ }^{51}$ C. R. Tolle, T. R. McJunkin, and D. J. Gorsich, IEEE Trans. Pattern Anal. Mach. Intell. 25, 32 (2003).

${ }^{52}$ D. L. Turcotte, J. Geophys. Res. 91, 1921, https://doi.org/10.1029/ JB091iB02p01921 (1986). 